\title{
Deciphering Citations Organized by Subject (Dcos) Index
}

Ivan Díaz, Martí Cortey, Àlex Olvera, Joaquim Segalés

\section{Source}

Ivan Díaz, Martí Cortey, Àlex Olvera, Joaquim Segalés. (2016). Use of H-Index and Other

Bibliometric Indicators to Evaluate Research Productivity Outcome on Swine Diseases.

PLOS ONE, vol. 11 (3), e0149690.

Dcos index would be defined as the number of publications that a certain author, institute or country (among others) holds within the set of papers included in the $\mathrm{H}$-index of a given area or subject. 